\title{
AhR and IDO1 in pathogenesis of Covid-19 and the "Systemic AhR Activation Syndrome:" a translational review and therapeutic perspectives
}

\author{
Waldemar A. Turski ${ }^{\mathrm{a}, *}$, Artur Wnorowski ${ }^{\mathrm{b}}$, Gabrielle N. Turskic,d, \\ Christopher A. Turski ${ }^{\mathrm{c}, \mathrm{d}}$ and Lechoslaw Turski ${ }^{\mathrm{d}}$ \\ ${ }^{a}$ Department of Experimental and Clinical Pharmacology, Medical University, Lublin, Poland \\ ${ }^{\mathrm{b}}$ Department of Biopharmacy, Faculty of Pharmacy, Medical University, Lublin, Poland \\ ${ }^{\mathrm{c}}$ Department of Ophthalmology, University of Bonn, Bonn, Germany \\ ${ }^{\mathrm{d}}$ German Center for Neurodegenerative Diseases, Bonn, Germany
}

\begin{abstract}
Covid-19 is the acute illness caused by SARS-CoV-2 with initial clinical symptoms such as cough, fever, malaise, headache, and anosmia. After entry into cells, corona viruses $(\mathrm{CoV})$ activate aryl hydrocarbon receptors (AhRs) by an indoleamine 2,3-dioxygenase (IDO1)-independent mechanism, bypassing the IDO1-kynurenine-AhR pathway. The IDO1kynurenine-AhR signaling pathway is used by multiple viral, microbial and parasitic pathogens to activate AhRs and to establish infections. AhRs enhance their own activity through an IDO1-AhR-IDO1 positive feedback loop prolonging activation induced by pathogens. Direct activation of AhRs by $\mathrm{CoV}$ induces immediate and simultaneous up-regulation of diverse AhR-dependent downstream effectors, and this, in turn, results in a "Systemic AhR Activation Syndrome" (SAAS) consisting of inflammation, thromboembolism, and fibrosis, culminating in multiple organ injuries, and death. Activation of AhRs by $\mathrm{CoV}$ may lead to diverse sets of phenotypic disease pictures depending on time after infection, overall state of health, hormonal balance, age, gender, comorbidities, but also diet and environmental factors modulating AhRs. We hypothesize that elimination of factors known to up-regulate AhRs, or implementation of measures known to down-regulate AhRs, should decrease severity of infection. Although therapies selectively down-regulating both AhR and IDO1 are currently lacking, medications in clinical use such as dexamethasone may down-regulate both AhR and IDO1 genes, as calcitriol/vitamin $\mathrm{D}_{3}$ may down-regulate the AhR gene, and tocopherol/vitamin E may down-regulate the IDO1 gene. Supplementation of calcitriol should therefore be subjected to epidemiological studies and tested in prospective trials for prevention of CoV infections, as should tocopherol, whereas dexamethasone could be tried in interventional trials. Because lack of physical exercise activates AhRs via the IDO1-kynurenine-AhR signaling pathway increasing risk of infection, physical exercise should be encouraged during quarantines and stay-at-home orders during pandemic outbreaks. Understanding which factors affect gene expression of both AhR and IDO1 may help in designing therapies to prevent and treat humans suffering from Covid-19.
\end{abstract}

Keywords: Corona virus, SARS-CoV-2, Covid-19, aryl hydrocarbon receptor, indoleamine 2,3-dioxygenase, systemic AhR activation syndrome (SAAS), inflammation, thromboembolism, fibrosis, multiple organ injury, brain injury, respiratory failure, dexamethasone, vitamin $\mathrm{D}$, vitamin $\mathrm{E}$, physical exercise

\footnotetext{
${ }^{*}$ Corresponding author: Waldemar A. Turski, MD, PhD, Department of Experimental and Clinical Pharmacology, Medical University, Jaczewskiego 8b, 20-090 Lublin, Poland.
}

Tel.: +48 81448 6452, +48 814486457 ; E-mail: waldemar. turski@umlub.pl. 


\section{AhR and IDO1 signaling in pathogenesis of Covid-19}

To trigger Covid-19, SARS-CoV-2 enters cells via angiotensin-converting enzyme 2 (ACE-2) by means of activating viral spike glycoproteins (SARS-2-S) by transmembrane protease serine 2 (TMPRSS2) to engage them with the enzyme expressed in the surfactant secreting alveolar cells of the lung (Letko, Marzi, \& Munster, 2020; Li et al., 2003). How SARS-CoV2 infection translates into systems pathology known as Covid-19 remains unclear. However, infection with murine corona viruses activates the $\mathrm{AhR}$ in an IDO1-independent manner, up-regulating expression of proviral TCDD-inducible-PARP (TiPARP) and modulating cytokines (Grunewald, Shaban, Mackin, Fehr, \& Perlman, 2020).

$\mathrm{AhR}$ is a nuclear receptor playing a critical role in immune and inflammatory processes and in modulating responses to the environment (Veldhoen et al., 2008). AhRs are kept in cytoplasm by chaperones Hsp90 (heat shock protein 90) and XAP (X-associated protein 2) and are transported into the nucleus after binding ligands to heterodimerize with ARNT (aryl hydrocarbon receptor nuclear translocator), and to bind to DNA to regulate gene expression. IDO1 is a rate-limiting enzyme converting tryptophan to kynurenine, which is an endogenous ligand activating AhR. Inflammatory factors, such as tumor necrosis factor- $\alpha(\mathrm{TNF} \alpha)$ and interleukins activate IDO1, whereas AhR also enhance their own activity through activation of a IDO1-AhR-IDO1 positive feedback loop prolonging the effects of AhR activation by other factors, including pathogens (Larigot, Jurice, Dairou, \& Coumoul, 2018; Neavin, Liu, Ray, \& Weinshilboum, 2018).

AhR can bind many diverse ligands including exogenous natural and synthetic, and endogenous chemicals (Stockinger, Di Meglio, Gialitakis, \& Duarte, 2014; Stockinger, Hirota, Duarte, \& Veldhoen, 2011; Wheeler, Rothhammer, \& Quintana, 2017). Effects of clinically used drugs on gene expression mediated by AhR and IDO1 in humans are hardly known.

\section{Inflammation may be $A h R$ dependent}

Corona viruses (CoV) activate $\mathrm{AhR}$ by an IDO1-independent mechanism, bypassing the IDO1kynurenine-AhR pathway, leading to mostly unrestricted up-regulation of downstream effectors such

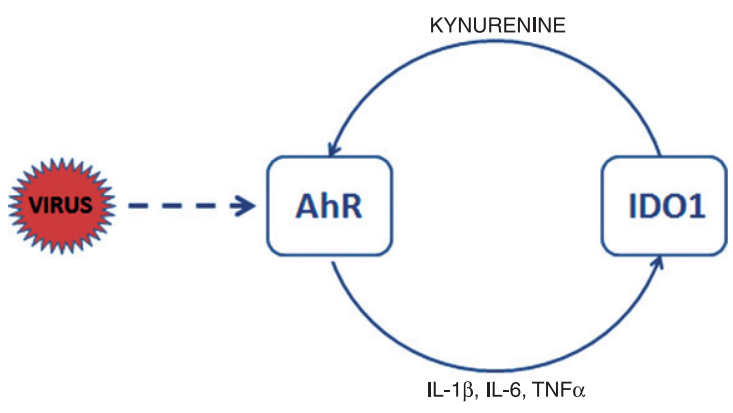

Fig. 1. AhR activation in CoV-infected cells. CoV activate AhR by means of AhR-activating ligand independent of IDO1. Upon activation AhR translocates to the nucleus to bind to genomic DNA and to generate downstream effectors such as AhRR, CYPs, TiPARP, and cytokines. IDO1 is induced by inflammatory factors, such as TNF $\alpha$ and interleukins 6 (IL-6) and $1 \beta$ (IL-1 $\beta$ ). AhR also enhances its own activity through activation of IDO1-AhR-IDO1 positive feedback loop prolonging the effects of AhR activation by other pathways. Activation of IDO1 leads in immune cells to release of kynurenine, a tryptophan metabolite, which is an endogenous ligand activating AhR. Exogenous ligands binding to AhR are dioxins such as TCDD.

as pro viral factor TiPARP, and to the modulation of cytokine gene expression, specifically, interleukin $1 \beta$ (IL-1 $\beta$ ), IL-10, and TNF- $\alpha$ (Fig. 1), which is consistent with the role for AhR activation in the host response to $\mathrm{CoV}$ infection (Chen et al., 2020; Grunewald, Shaban, Mackin, Fehr, \& Perlman, 2020; Neavin, Liu, Ray, \& Weinshilboum, 2018). This ability of $\mathrm{CoV}$ allows other pathogens and comorbidities to enhance intensity of its infection by activating AhR via an IDO1-dependent mechanism, also leading to inflammation. On the other hand, this ability to activate AhR by an IDO1-independent mechanism prevents $\mathrm{CoV}$-induced infection from activating host immune-suppressive effects which depend on kynurenines to limit inflammation (Jaronen, \& Quintana, 2014). When CoV infection persists, inflammation activates IDO1 by massively releasing cytokines (Chen et al., 2019; Larigot, Jurice, Dairou, \& Coumoul, 2018; Neavin, Liu, Ray, \& Weinshilboum, 2018). This, in turn, perpetuates an already extensive viral activation of AhR by additional release of kynurenine. At that time self-limiting control mechanisms of the host immune response may derail due to intensity of AhR activation by multiple acting mechanisms, and a cytokine deluge may begin (Fig. 2). 


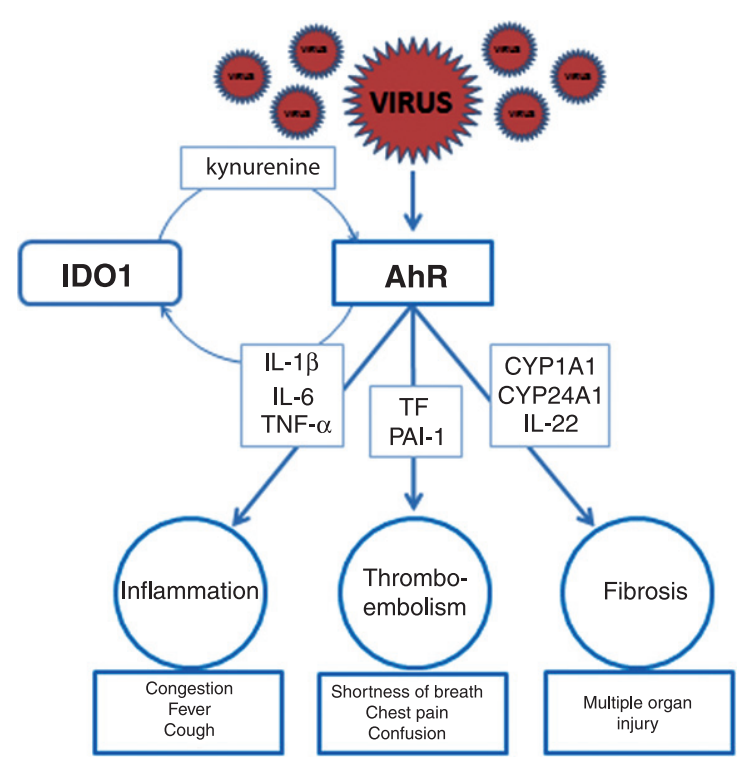

Fig. 2. Activation of AhR signaling pathways contributing to pathogenesis of $\mathrm{CoV}$ infections: a hypothesis. To induce inflammation (left upper panel), CoV activate AhR by an IDO1-independent mechanism resulting in up-regulation of downstream effectors such as TiPARP (TCDD-inducible-PARP), cytokines including interleukin $1 \beta$ (IL-1 $\beta$ ) and IL-6, and tumor necrosis factor alpha $(\mathrm{TNF}-\alpha)$. Characteristic clinical symptoms of early inflammation are congestion, fever and cough (left lower panel) complemented by fatigue, headache, anosmia, muscle aches, sore throat, nausea, diarrhea, and skin rashes. To induce thromboembolism (middle upper panel), another set of downstream effectors such as TF (tissue factor) and PAI-1 (plasminogen activator inhibitor 1) need to be up-regulated. Key clinical symptoms possibly indicating pulmonary, cardiovascular, or neurological complications are shortness of breath, chest pain, and confusion (middle lower panel) often accompanied by bluish lips and inability to wake. In addition, fibrosis with multiple organ injury develops when AhR activates CYP1A1/IL-22 signaling pathway with STAT3 (signal transducer and activator of transcription 3) promoting fibrogenesis and fibrosis (right upper panel). Critical factors allowing early diagnosis are specific tissue biomarkers indicating organ damage such as troponin for the heart. In critically ill patients infected with $\mathrm{CoV}$ multiple AhR signaling pathways may be activated simultaneously resulting in the systemic AhR activation syndrome (SAAS).

\section{Thromboembolism may be AhR dependent}

Critically ill patients infected with SARS-CoV-2 develop severe multisystem inflammatory syndrome consisting of respiratory distress, cardiovascular dysfunction, thrombosis, neurological manifestations, dysregulated inflammation, and fibrosis (Leisman, Deutschman, \& Legrand, 2020; Oxley et al., 2020). Clinical experience is teaching us that individuals with SARS-CoV-2 infections are at high risk of fatal thromboembolism (Wichmann et al., 2020) including the brain (Oxley et al., 2020). How SARS-CoV-2 infection can trigger thromboembolism also in young and formerly healthy humans is not understood. However, since $\mathrm{CoV}$ activates $\mathrm{AhR}$, it may also lead to up-regulation of downstream effectors such as tissue factor (TF) and plasminogen activator inhibitor 1 (PAI-1) in endothelial cells, consistent with the role for AhR activation of AhR-TF/PAI-1 axis as a part of the host response to $\mathrm{CoV}$ infection (Belghasem et al., 2019). If the inflammatory vicious circle due to cytokine elevation persists and the IDO1-kynurenineAhR pathway becomes activated, the intensity of AhR activation may increase and thromboembolism may occur in different organs (Fig. 2).

\section{Fibrosis may be AhR dependent}

Individuals suffering from SARS-CoV-2 infections are also at high risk of multiple organ fibrosis including the lung, heart, kidney, and gastrointestinal tract organs (Babapoor-Farrokhran et al., 2020; Ye, Zhang, Wang, Huang, \& Song, 2020). Although AhR mainly contributes to metabolism of xenobiotics, it also regulates expression of genes involved in fibrogenesis (Stevens, Mezrich, \& Bradfield, 2009). After binding ligands, AhRs heterodimerize with ARNT, and are transported into the nucleus to bind with xenobiotic response elements (XREs) in promoter regions of the target genes including CYP1A1, CYP24A1, and IL-22 (Weidenbusch et al., 2017). Subsequently, IL-22 binds to its receptor and after intermediate steps, phosphorylated STAT3 (signal transducer and activator of transcription 3) acts as a transcription factor for AhR/IL-22 signaling pathway (Weidenbusch et al., 2017) and dysregulated fibrogenesis in multiple organs can begin, provided activation of AhRs persists (Fig. 2).

\section{Preterm birth may be AhR dependent}

Most common adverse pregnancy outcome during the first two trimesters in women infected with $\mathrm{CoV}$ is preterm birth (Di Mascio et al., 2020). Miscarriage, cesarean delivery, and perinatal death may occur more often, too (Di Mascio et al., 2020). When infection manifests during the third trimester of pregnancy, such adverse outcomes may not occur (Yan et al., 2020). AhR is densely expressed in the human placenta and the AhR gene is up-regulated in placentas of women suffering from unexplained miscarriage (Fan, Su, Yang, \& Zhao, 2017). In that context, activation 
of placental AhRs by $\mathrm{CoV}$ can trigger release of cytokines by placenta and fetus leading to activation of cyclooxygenase-2 (COX2) in trophoblasts and amniotic epithelial cells and release of prostaglandins such as PGE2 and PGF2 $\alpha$ (Li et al., 2015). We hypothesize that if the activation of AhRs during $\mathrm{CoV}$ infection persists, activation of AhR-COX2/PG pathway may, in sensitive individuals, initiate myometrial contractions and lead to preterm birth (Li et al., 2015).

\section{Multiple organ injury may be AhR dependent}

Protracted infection with SARS-CoV-2 may lead to injuries of multiple organs including the lung, heart, kidney, and liver (Zheng, Ma, Zhang, \& Xie, 2020). CoV infected children can also develop such multiple organ injuries (Cui et al., 2020). During early infection, translocation of SARS-CoV-2/ACE-2 complexes into the lung alveolar epithelial cells, or other organ epithelial cells, and massive intracellular viral replication (Cava et al., 2020) may lead to cell death (Chen, Li, Chen, Feng, \& Xiong, 2020) due to activation of AhRs leading to increased expression of CYP1 family genes such as CYP1A1, CYP1B1, and CYP1A2. This results in initiation of apoptosis via activation of caspases dependent pathways, and to ACE-2 down-regulation, which after release of viruses from infected host cells offers a new entry establishing a multiple positive feedback loop accelerating infection (Abassi, Assady, Khoury, \& Heyman, 2020). During late phase of the disease, cytokine elevation related to persistent activation of AhRs by $\mathrm{CoV}$, can also induce tissue injury in diverse organs, and contribute to worsening of clinical manifestations and disease outcomes. Cardiomyocytes exposed to TCDD, an AhR agonist, also respond with increasing CYP1A1 mRNA and protein expression in an AhR dependent manner suggesting activation of the AhR/CYP1A1 signaling pathway (Zhou et al., 2017). Therefore, we hypothesize, that the AhR/CYP1 signaling pathway may be activated by $\mathrm{CoV}$ in organs undergoing tissue injuries during both early and late stages of disease, and that elevated cytokines such as IL-6, IL-8 or IL-1ß (Leisman, Deutschman, \& Legrand, 2020) may activate diverse signal cascades causing and accelerating tissue injury. One such mechanism involves the Notch-AhR-IL22 pathway regulating tissue homeostasis (Weidenbusch et al., 2017). In macrophages, Notch signaling enables production of
IL-6, which in turn amplifies Notch-dependent signaling establishing another positive feedback loop accelerating synthesis of IL-6 and perpetuating the Notch-AhR-IL-22 signaling pathway (Weidenbusch et al., 2017). If the inflammatory deluge due to cytokine elevation persists, multiple positive feedback loops become activated whereas the inhibitory feedback loops become gradually inactive. Then the intensity of AhR activation may further increase and tissue damage may occur in different organs. In addition, AhR controls organ homeostasis by governing epithelial cell networks such as intestinal, alveolar, bronchial, or tubular, providing a barrier against toxins and pathogens, by supporting their integrity by means of preventing malignant transformation and allowing for their timely renewal (Rothhammer, \& Quintana, 2019). In intestine, activation of AhR by IDO1 induced by oxazoles can suppress anti-inflammatory action of IL-10 and lead to inflammation mediated by IL-13 and IFN $\gamma$-producing killer T cells (Iyer et al., 2018). Whether such a mechanism can be activated by viral activation of IDO1 or AhR or both, and whether it may be functional in organs other than intestine, is not known.

\section{Brain injury may be AhR dependent}

Changes in smell and taste perceptions belong to the earliest symptoms indicating viral invasion of the nervous system by $\mathrm{CoV}$ in humans (Lechien et al., 2020; Zubair et al., 2020). However, olfactory sensory neurons do not express either ACE-2 or related serine protease TMPRSS2 genes allowing SARS-CoV-2 passage through neuronal membranes (Brann, Tsukahara, Weinreb, Logan, \& Datta, 2020). Since CoV including SARS-CoV-2 enter neurons and may induce brain injury in humans (Zhou, Zhang, Wang, \& Gao, 2020), and since in transgenic mice lacking ACE-2, SARS-CoV-1 can enter the brain via olfactory bulbs and spread transsynaptically over many days towards interconnected cortical subfields, amygdala, hippocampus, thalamus, midbrain, periaqueductal gray, and pons causing neuronal death (Netland et al., 2008), we suspect an alternative non-ACE-2 dependent entry mechanism allowing neuronal invasion by $\mathrm{CoV}$. When neuronal entry is established, SARS-CoV-2 will activate AhRs, as it does in non-neuronal cell populations, thereby increasing gene expression of the CYP1 family such as CYP1A1, CYP1B1, and CYP1A2 and initiate apoptosis via activation of caspases (Rzemieniec 
et al., 2019), resulting in progressive death of neurons and brain injury (Zhou, Zhang, Wang, \& Gao, 2020). It is intriguing that mechanisms involving activation of $A h R$ in neurons leading to apoptotic brain injury have been confirmed in experimental models of stroke (Cuartero et al., 2014).

The brain is complex compared to other organs thereby preventing neurotropic viruses from invading it all at once due to morphological organization and cellular diversity, long distances between different neuronal populations, and synaptic traps set between interconnected parts of it. As the silent and slow journey of $\mathrm{CoV}$ through the brain towards the respiratory center continues, cell damage accumulating in initially affected neuronal populations can influence sensitivity of next targeted neuronal populations. Since brain injury reported by Netland et al. (2008) in SARS-CoV-1 infected mice was similar to that triggering spontaneous focal seizures in rats (Cavalheiro et al., 1991), we hypothesize that brain injury cumulating over time in infected individuals may lead to silent limbic seizures, or induce seizure threshold changes. It is intriguing, that even overt convulsions can occur in Covid-19 patients (Hepburn et al., 2020) and that careful analyses of cohorts of critically ill patients suffering from Covid19 are confirming such observations (Roman et al., 2020), which, however, are still rare and require more attention during follow-up studies. Brain stimulation studies in humans indicate that neuronal limbic/paralimbic networks, which are targeted by infection with $\mathrm{CoV}$ in mice (Netland et al. 2008), and consist of amygdaloid and hippocampal neuronal networks, drive in humans ictal central apnea by affecting the periaqueductal gray (Lacuey, Hampson, Harper, Miller, \& Lhatoo, 2019). The periaqueductal gray is responsible for modulation of pre-Bötzinger complex neurons, which control the eupneic respiratory rhythm (Subramanian, \& Holstege, 2013). Since periaqueductal gray is targeted by CoV (Netland et al., 2008) and it may also be affected by a dysfunctional limbic/paralimbic breathing modulation network in humans (Lacuey, Hampson, Harper, Miller, \& Lhatoo, 2019), we hypothesize that in critically ill patients $\mathrm{CoV}$ may reach the medulla and the pre-Bötzinger complex, which contains respiration regulating cholinergic neurons (Carey, Dunn, \& Gaspari, 2013), and contribute to their activation, apnea, and death. Stimulation of the caudal part of the ventrolateral periaqueductal gray in rats inhibits both pre-inspiratory cells and the diaphragm leading to respiratory failure lacking dyspnea (Subramanian
\& Holstege, 2013). If CoV reach periaqueductal gray and enter cholinergic neurons in the pre-Bötzinger complex, activation of AhR may also suppress activity of acetylcholinesterase (AChE) in these neurons (Xie et al., 2013) and induce stimulation of effector cells, eventually leading to inhibition of preinspiratory cells and apnea.

\section{Environment and AhR}

Early analyses indicate that exposure to air pollutants may complicate the course of Covid-19 and lead to more severe and lethal outcomes (Domingoa, \& Rovira, 2020). Atmospheric particulate matter air pollutants such as polycyclic aromatic hydrocarbons and dioxins, but also nitrogen oxide, ozone, and carbon monoxide have deleterious effects on the respiratory system in humans, and AhR is a target for several of them (O'Driscoll, \& Mezrich, 2018). Individuals chronically exposed to such pollutants may be at higher risk of contracting viral infections since AhRs determine innate and adaptive immunity, and govern transcription of xenobiotic metabolizing enzymes such as cytochrome P450 with CYP1A1 and CYP1B1 (Tsatsakis et al., 2020). Since AhR signaling is dependent on multiple proteins, activation of AhRs by urban dust, polycyclic aromatic hydrocarbons, or diesel exhaust may lead to up-regulation of multiple sets of downstream effectors promoting different pathologies and making humans more prone to pathogens including CoV (Tsatsakis et al., 2020).

Another set of issues arises from clinical experience with SARS-CoV-2 indicating that severity of the disease in humans may vary from minimal to critically ill and disease duration from few days to many weeks. Assuming that during clinically active Covid-19 AhRs remain maximally activated poses the question of long-term sequelae of Covid-19, which needs to be addressed by epidemiological studies in survivors. Relevant clues guiding towards answers may arrive from epidemiological studies on human exposure to the Agent Orange contaminated with dioxin TCDD, which is an AhR agonist, pointing to increased prevalence of cancers, endocrine disorders, neurological disorders, pulmonary diseases, and liver cirrhosis (Yi, Hong, Ohrr, \& Yi, 2014).

Since $\mathrm{CoV}$ persistently activate AhRs, this may lead to up-regulation of multiple sets of downstream effectors resulting in different pathologies (Fig. 2) depending on time after infection, individuals overall state of health, comorbidities, and environmental 
factors affecting AhRs. We believe it is therefore appropriate to describe this disease as a systemic AhR activation syndrome (SAAS), which can manifest in an acute (current pandemic), and perhaps later, in a chronic form, in survivors.

\section{Dual mechanism of $\mathrm{AhR}$ activation: a chance for therapy}

Since CoV initially bypass the common IDO1kynurenine-AhR pathway, factors which activate AhR directly may facilitate infection. Eliminating such factors may be critical for prophylaxis but also for therapy of $\mathrm{CoV}$ infections. When $\mathrm{CoV}$ enter the human body and symptoms are mild or disease is subclinical (Huang et al., 2020; Xu et al., 2020), we hypothesize that $\mathrm{CoV}$ activate AhR, but the IDO1kynurenine-AhR pathway may be inactive (Fig. 1). If so, the fate of viral infection may depend on the state of background AhR activation, which is determined by environmental pollutants, gender, age, diet, physical activity, and comorbidities. We hypothesize that as long as AhR remains activated due to CoV infection and the symptoms are mild, eliminating factors known to up-regulate AhR, or implementing means known to down-regulate AhR should decrease severity of infection. When infection by $\mathrm{CoV}$ has been fully established, and physicians care for patients suffering from severe symptoms (Huang et al., 2020; Oxley et al., 2020; Xu et al., 2020), we hypothesize that the IDO1-kynurenine-AhR pathway is continuously activated due to protracted inflammation in addition to $\mathrm{CoV}$ mediated activation of AhR. Such a vicious circle can only be efficiently interrupted by simultaneous down-regulation of both AhR and IDO1 (Fig. 1). A medication addressing activity of both AhR and IDO1 simultaneously is dexamethasone (Ott et al., 2015; Vrzal et al., 2009), a glucocorticoid with a broad range of uses including asthma, chronic obstructive pulmonary disease, and rheumatoid arthritis.

\section{AhR alone}

AhR was discovered as a receptor binding and mediating toxicity of the environmental dioxin TCDD (2,3,7,8-tetrachlorodibenzo-p-dioxin) (Okey, 2007; Opitz et al., 2011). This initial observation may indicate that humans frequently exposed to environmental and other toxins activating AhR such as dioxins or pesticides may be more prone to, and at risk of, contracting infection due to $\mathrm{CoV}$ more easily. Surprisingly, calcitriol (vitamin $\mathrm{D}_{3}$ ) in addition to its main mechanisms of action, may down-regulate expression of the AhR gene (Takami, Fujimaki, Nishimura, \& Iwashima, 2015). More surprisingly, deficiency of vitamin $\mathrm{D}_{3}$ has indeed been reported to contribute to acute respiratory distress syndrome with fatalities increasing with age and comorbidities, both being associated with lower concentrations of vitamin D (Grant et al., 2020). The concentration of vitamin $\mathrm{D}$ decreases with age leaving the elderly particularly prone to viral infections including SARS-CoV-2, which is reflected in the pattern of Covid-19 during the current pandemic. If confirmed in epidemiological studies, supplementation of vitamin D during winter should be recommended to all, and particularly in the elderly since vitamin D may down-regulate the AhR gene (Takami, Fujimaki, Nishimura, \& Iwashima, 2015), therefore decreasing susceptibility to viral infections.

\section{IDO1 alone}

IDO1 is a cytosolic, rate-limiting enzyme in the conversion of tryptophan, via kynurenine, which is a ligand of and activates AhR (Jaronen, \& Quintana, 2014), towards synthesis of nicotinamide (Mellor, Lemos, \& Huang, 2017). Activation of IDO1 depresses $\mathrm{T}$ lymphocytes activation by starving them of tryptophan through its conversion to kynurenine (Neavin, Liu, Ray, \& Weinshilboum, 2018). IDO1 gene transcription can be up-regulated by multiple viral, microbial and parasitic pathogens, cancers, or pregnancy (Benavente et al., 2019; Larigot, Juricek, Dairou, \& Coumoul, 2018; Neavin, Liu, Ray, \& Weinshilboum, 2018) or it can be induced by the proinflammatory cytokine INF- $\gamma$ (Neavin, Liu, Ray, \& Weinshilboum, 2018) limiting host immune response by means of generation of FOXP3(forkhead box P3)expressing regulatory $\mathrm{T}$ cells to inhibit other cells of the immune system (Larigot, Juricek, Dairou, \& Coumoul, 2018; Neavin, Liu, Ray, \& Weinshilboum, 2018).

The most popular drug down-regulating IDO1 is tocopherol/vitamin E (Stapelberg et al., 2013). Vita$\min \mathrm{E}$ is an antioxidant that scavenges radicals, terminates oxidation of polyunsaturated fatty acids, and has multiple immunomodulatory actions such as on lymphocyte proliferation, prostaglandin E2 synthesis, affects gene transcription and translation, and it influences cell membrane and signal transduction 
(Lee, \& Han, 2018). It is intriguing that up to $60 \%$ of adults in the United States have too low vitamin E intake ( $<12 \mathrm{mg} / \mathrm{d}$ ) (Fulgoni, Keast, Bailey, \& Dwyer, 2011). Drawbacks of such a low intake are dysregulation of immune and inflammatory responses and decreased resistance to infections with elderly humans being hit the most. This is due to the fact that aging pathogen defense-related immune responses are weaker and inflammatory responses prolonged (Meydani, Lewis, \& Wu, 2018). It is therefore conceivable, that insufficient supply with vitamin E may contribute to increased susceptibility in the elderly to infection with $\mathrm{CoV}$ and may promote severity of Covid-19. Supplementation of vitamin E in elderly could reduce the risk of, and morbidity from, infections with $\mathrm{CoV}$. This has intuitively been recognized in Great Britain where Covid-19 patients were supplemented with vitamins (Calder, Carr, Gombart, \& Eggersdorfer, 2020).

\section{Habits may modulate $A h R$}

In healthy conditions, tryptophan is metabolized to kynurenine by tryptophan-2,3-dioxygenase (TDO) in the liver and by IDO1 in the brain and peripheral tissues such as small intestine, lungs, female genital tract or placenta (Badawy, 2017; Castro-Portuguez, \& Sutphin, 2020; Sedlmayr, 2007). In addition, significant amount of kynurenine is metabolized by exercising muscles (Agudelo et al., 2019; Martin, Azzolini, \& Lira Ruas, 2020) and a small amount is used to activate AhR in response to minor inflammatory processes (Badawy, 2017). Disrupting this healthy balance by immobility such as through stayat-home orders, may lead to an increase in kynurenine and its availability to activate AhR, therefore increasing an individual's risk to become prone to infection (Agudelo, 2019; Martin, Azzolini, \& Lira Ruas, 2020). Therefore, physical exercise during quarantine should be encouraged.

In advanced disease, if liver function decreases and ceases (Zhang, Shi, \& Wang, 2020) and muscles are not stimulated due to immobilization in the hospital, AhR may become overwhelmed with kynurenines and activated via the IDO1-kynurenine-AhR pathway. If such patients are subjected to intensive therapy in the ICUs, principles of care established to excel under regular conditions should perhaps be expanded by following biomarkers of tryptophan metabolism related to inflammation (Badawy, \& Guillemin, 2019).

\section{Lessons to learn}

Persistent activation of AhRs by $\mathrm{CoV}$ may lead to up-regulation of multiple sets of downstream effectors governed by AhRs resulting in different phenotypic disease pictures depending on time after infection, individuals overall state of health, comorbidities, and environmental and other factors modulating AhRs.

$\mathrm{CoV}$ are perfect viruses invading hosts by avoiding collision with other pathogens because of activating the AhR when bypassing the IDO1-kynurenine-AhR pathway. This pathway is employed by multiple, viral, microbial and parasitic pathogens to establish infection. This peculiarity enables massive upregulation of multiple AhR-dependent downstream effectors, whereas self-restricting mechanisms may remain at bay.

Therapies selectively down-regulating both AhR and IDO1 are currently lacking and should be considered in the future by public and private innovation driven research organizations as therapies for viral infections. However, such therapies should be designed to leave physiological functions of both AhRs and IDO1 intact. Since dexamethasone may down-regulate both AhR and IDO1 genes, in addition to its main mechanism of action, and is known to play a role in inflammatory diseases, its use in therapy of Covid-19 should be studied in rigorous interventional trials.

Since calcitriol may down-regulate the AhR gene and is known to play a role in the spread of other viral infections, supplementation of calcitriol should be reviewed in epidemiological studies and tested in prospective trials for prevention of SARS-CoV-2 infections.

Supplementation of tocopherol should be reviewed in epidemiological studies and tested in prospective trials for prevention of SARS-CoV-2 infections in elderly since tocopherol may down-regulate IDO1 gene and is known to play a role in response to viral infections and inflammation in aging.

Associations and conclusions revealed by our analyses should be considered with particular caution because we are reporting changes in the expression of genes only. Such changes need to be translated into biological actions by multiple associated mechanisms, which have not been evaluated in our analyses. Therefore, our hypotheses offer no information or confirmation of clinical therapeutic value of any discussed therapy in modulating of SARS$\mathrm{CoV}-2$ infection. Before any conclusion can be drawn 
regarding a potential benefit of our recommendations regarding any medication in patients with Covid-19 randomized controlled clinical trials and large observational population studies evaluating our concepts will be necessary.

\section{References}

Abassi, Z., Assady, S., Khoury, E.E., \& Heyman, S.N. (2020). Angiotensin-converting enzyme 2: an ally or a Trojan horse? Implications to SARSCoV-2-related cardiovascular complications. American Journal of Physiology Heart and Circulation Physiology, 318(5), H1080-H1083, doi:10.1152/ajpheart.00215.2020.

Agudelo, L.Z., Ferreira, D.M.S., Dadvar, S., Cervenka, I., Ketscher, L., Izadi, M., Zhengye, L., Furrer, R., Handschin, C., Venckunas, T., Brazaitis, M., Kamandulis, S., Lanner, J.T., \& Ruas, J.L. (2019). Skeletal muscle PGC-1 $\alpha 1$ reroutes kynurenine metabolism to increase energy efficiency and fatigue-resistance. Nature Communications, 10, 2767. doi: 10.1038/s41467-019-10712-0.

Babapoor-Farrokhran, S., Gill, D., Walker, J., Rasekhi, R.T., Bozorgnia, B., \& Amanullah, A. (2020). Myocardial injury and COVID-19: Possible mechanisms. Life Sciences, 253(117723). doi:10.1016/j.lfs.2020.117723.

Badawy, A.A.-B. (2017). Kynurenine pathway of tryptophan metabolism: Regulatory and functional aspects. International Journal of Tryptophan Research, 10(1178646917691938). doi:10.1177/1178646917691938.

Badawy, A.A.-B., \& Guillemin, G. (2019). The plasma [kynurenine]/[tryptophan] ratio and indoleamine 2,3dioxygenase: time for appraisal. International Journal of Tryptophan Research, 12(1178646919868978). doi:10.1177/ 1178646919868978.

Belghasem, M., Roth, D., Richards, S., Napolene, M.A., Walker, J., Yin, W., Arinze, N., Lyle, C., Spencer, C., Francis, J.M., Thompson, C., Andry, C., Whelan, S.A., Lee, N., Ravid, K., \& Chitalia, V.C. (2019). Metabolites in a mouse cancer model enhance venous thrombogenicity through the aryl hydrocarbon receptor-tissue factor axis. Blood, 134(26), 2399-413. doi:10.1182/blood.2019001675.

Benavente, F.M., Soto, J.A., Pizarro-Ortega, M.S., Bohmwald, K., González, P.A., Bueno, S.M., \& Kalergis, A.M. (2019). Contribution of IDO to human respiratory syncytial virus infection. Journal of Leukocyte Biology, 106(4), 933-42. doi:10.1002/JLB.4RU0219-051RR.

Brann, D., Tsukahara, T., Weinreb, C., Logan, D.W., \& Datta, S.R. (2020). Non-neural expression of SARS-CoV-2 entry genes in the olfactory epithelium suggests mechanisms underlying anosmia in COVID-19 patients. BioRxiv. doi:org/10.1101/2020.03.25.009084.

Calder, P.C., Carr, A.C., Gombart, A.F., \& Eggersdorfer, M. (2020). Optimal nutritional status for a well-functioning immune system is an important factor to protect against viral infections. Nutrients, 12(4). doi:10.3390/nu12041181.

Carey, J.L., Dunn, C., \& Gaspari, R.J. (2013). Central respiratory failure during acute organophosphate poisoning. Respiratory Physiology \& Neurobiology, 189, 403-410. doi:org/10.1016/j.resp.2013.07.022.
Castro-Portuguez, R., \& Sutphin, G.L. (2020). Kynurenine pathway, $\mathrm{NAD}^{+}$synthesis, and mitochondrial function: Targeting tryptophan metabolism to promote longevity and healthspan. Experimental Gerontology, 132(110841). doi: 10.1016/j.exger.2020.110841.

Cava, C., Bertoli, G., \& Castiglioni, I. (2020). In silico discovery of candidate drugs against Covid-19. Viruses, 12(4), 404. doi:10.3390/v12040404

Chen, G., Wu, D., Guo, W., Cao, Y., Huang, D., Wang, H., Wang, T., Zhang, X., Chen, H., Yu, H., Zhang, X., Zhang, M., Wu, S., Song, J., Chen, T., Han, M., Li, S., Luo, X., Zhao, J., \& Ning, Q. (2020). Clinical and immunological features of severe and moderate coronavirus disease 2019. Journal of Clinical Investigation, 130(5), 2620-9. doi:10.1172/JCI137244.

Chen, L., Li, X., Chen, M., Feng, Y., \& Xiong, C. (2020). The ACE-2 expression in human heart indicates new potential mechanism of heart injury among patients infected with SARS-CoV-2. Cardiovascular Research, 116(6). doi: $10.1093 / \mathrm{cvr} / \mathrm{cvaa} 078$.

Cuartero, M.I., Ballesteros, I., de la Parra, J., Harkin, A.L., Abautret-Daly, A., Sherwin, E., Fernández-Salguero, P., Corbí, Á.L., Lizasoain, I., \& Moro, M.A. (2014). LKynurenine/Aryl hydrocarbon receptor pathway mediates brain damage after experimental stroke. Circulation, 130, 2040-51. doi: 10.1161/CIRCULATIONAHA.114.011394.

Cui, Y., Tian, M., Huang, D., Wang, X., Huang, Y., Fan, L., Wang, L., Chen, Y., Liu, W., Zhang, K., Wu, Y., Yang, Z., Tao, J., Feng, J., Liu, K., Ye, X., Wang, R., Zhang, X., \& Zha, Y. (2020). A 55-day-old female infant infected with 2019 novel coronavirus disease: presenting with pneumonia, liver injury, and heart damage. Journal of Infectious Diseases, 221(11), 1775-81. doi:10.1093/infdis/jiaa113.

Di Mascio, D., Khalil, A., Saccone, G., Rizzo, G., Buca, D., Liberati, M., Vecchiet, J., Nappi, L., Scambia, G., Berghella, V., \& D'Antonio, F. (2020). Outcome of coronavirus spectrum infections (SARS, MERS, COVID-19) during pregnancy: a systematic review and meta-analysis. American Journal of Obstetrics \& Gynecology, 100107. doi: 10.1016/j.ajogmf.2020.100107.

Domingoa, J.L., \& Rovira, J. (2020). Effects of air pollutants on the transmission and severity of respiratory viral infections. Environmental Research, 187(109650). doi.org/10.1016/ j.envres.2020.109650.

Fan, H., Su, X., Yang, B., \& Zhao, A. (2017). Aryl hydrocarbon receptor and unexplained miscarriage. Journal of Obstetrics and Gynaecology Research, 43(6), 1029-36. doi: 10.1111/jog.13309.

Fulgoni, V.L., Keast, D.R., Bailey, R.L., \& Dwyer, J. (2011). Foods, fortificants, and supplements: where do Americans get their nutrients? Journal of Nutrition, 141(10), 1847-54. doi: $10.3945 /$ jn.111.142257.

Grant, W.B., Lahore, H., McDonnell, S.L., Baggerly, C.A., French, C.B., Aliano, J.L., \& Bhattoa, H.P. (2020). Evidence that vitamin D supplementation could reduce risk of influenza and COVID-19 infections and deaths. Nutrients, 12(4), 988. doi: 10.3390/nu12040988.

Grunewald, M.E., Shaban, M.G., Mackin, S.R., Fehr, A.R., \& Perlman, S. (2020). Murine coronavirus infection activates the aryl hydrocarbon receptor in an indoleamine 2,3-dioxygenase-independent manner, contributing to 
cytokine modulation and proviral TCDD-inducible-PARP expression. Journal of Virology, 94(3), e01743-19. doi: 10.1128/JVI.01743-19.

Hepburn, M., Mullaguri, N., George, P., Hantus, S., Punia, V., Bhimraj, A., \& Newey, C.R. (2020). Acute symptomatic seizures in critically ill patients with COVID-19: Is there an association? Neurocritical Care, 1-5. doi: 10.1007/s12028020-01006-1.

Huang, C., Wang, Y., Li, X., Ren, L., Zhao, J., Hu, Y., Zhang, L., Fan, G., Xu, J., Gu, X., Cheng, Z., Yu, T., Xia, J., Wie, Y., Wu, W., Xie, X., Yin, W., Li, H., Liu, M., Xiao, Y., Gao, H., Guo, L., Xie, J., Wang, G., Jiang, R., Gao, Z., Jin, Q., Wang, J., \& Cao, B. (2020). Clinical features of patients infected with 2019 novel coronavirus in Wuhan, China. Lancet, 395(10223), 497506. doi: 10.1016/S0140-6736(20)30183-5.

Iyer, S.S, Gensollen, T., Gandhi, A., Oh, S.F., Neves, J.F., Collin, F., Lavin, R., Serra, C., Glickman, J.,de Silva P.S.A., Sartor, R.B., Besra, G., Hauser, R., Maxwell, A., Llebaria, A., \& Blumberg, R.S. (2018). Dietary and microbial oxazoles induce intestinal inflammation by modulating aryl hydrocarbon receptor responses. Cell, 173(5), 1123-34.e11. doi: 10.1016/j.cell.2018.04.037.

Jaronen, M., \& Quintana, F.J. (2014). Immunological relevance of the coevolution of IDO1 and AHR. Frontiers in Immunology, 5(521). doi: 10.3389/fimmu.2014.00521.

Lacuey, N., Hampson, J.P., Harper, R.M., Miller, J.P., Lhatoo, S. (2019). Limbic and paralimbic structures driving ictal central apnea. Neurology, 92(7), e655-e669. doi: 10.1212/WNL.0000000000006920.

Larigot, L., Juricek, L., Dairou, J., Coumoul, X. (2018). AhR signaling pathways and regulatory functions. Biochimie Open, 7, 1-9. doi: 10.1016/j.biopen.2018.05.001.

Lechien, J.R., Chiesa-Estomba, C.M., De Siati, D.R., Horoi, M., Le Bon, S.D., Rodriguez, A., Dequanter, D., Blecic, S., El Afia, F., Distinguin, L., Chekkoury-Idrissi, Y., Hans, S., Delgado, I.L., Calvo-Henriquez, C., Lavigne, P., Falanga, C., Barillari, M.R., Cammaroto, G., Khalife, M., Leich, P., Souchay, C., Rossi, C., Journe, F., Hsieh, J., Edjlali, M., Carlier, R., Ris, L., Lovato, A., De Filippis, C., Coppee, F., Fakhry, N., Ayad, T., \& Saussez, S. (2020). Olfactory and gustatory dysfunctions as a clinical presentation of mild-to-moderate forms of the coronavirus disease (COVID-19): a multicenter European study. European Archives of Oto-Rhino-Laryngology, 1-11. doi: 10.1007/s00405-020-05965-1.

Lee, G.Y., \& Han, S.N. (2018). The role of vitamin E in immunity. Nutrients, 10(11), 1614. doi: 10.3390/nu10111614.

Leisman, D.E., Deutschman, C.S., \& Legrand, M. (2020). Facing COVID-19 in the ICU: vascular dysfunction, thrombosis, and dysregulated inflammation. Intensive Care Medicine, 28, 1-4. doi: 10.1007/s00134-020-06059-6.

Letko, M., Marzi, A., \& Munster, V. (2020). Functional assessment of cell entry and receptor usage for SARS-CoV-2 and other lineage B betacoronaviruses. Nature Microbiology, 5(4), 56269. doi: 10.1038/s41564-020-0688-y.

Li, J., Long, X., Zhu, C., Wang, H., Wang, T., Lin, Z., Li, J., \& Xiong, N. (2020). Olfactory dysfunction in recovered COVID-19 patients. Movement Disorders. doi: $10.1002 /$ mds. 28172 .

Li, W., Moore, M.J., Vasilieva, N., Sui, J., Wong, S.K., Berne, M.A., Somasundaran, M., Sullivan, J.L., Luzuriaga,
K., Greenough, T.C., Choe, H., \& Farzan, M. (2003). Angiotensin-converting enzyme 2 is a functional receptor for the SARS coronavirus. Nature, 426(6965), 450-54. doi: 10.1038 /nature 02145 .

Li, Y., Wang, K., Zou, Q-Y., Zhou, C., Magness, R.R., \& Zheng, J. (2015). A possible role of aryl hydrocarbon receptor in spontaneous preterm birth. Medical Hypotheses, 84(5), 494-7. doi: 10.1016/j.mehy.2015.02.001.

Martin, K.S., Azzolini, M, \& Lira Ruas, J. (2020). The kynurenine connection: how exercise shifts muscle tryptophan metabolism and affects energy homeostasis, the immune system, and the brain. American Journal of Physiology - Cell Physiology, 318(5), C818-830. doi: 10.1152/ajpcell.00580.2019.

Mellor, A.L., Lemos, H., \& Huang, L. (2017). Indoleamine 2,3dioxygenase and tolerance: Where are we now? Frontiers in Immunology, 8(1360). doi: 10.3389/fimmu.2017.01360.

Meydani, S.N., Lewis, E.D., Wu, D. (2018). Perspective: Should vitamin E recommendations for older adults be increased? Advances in Nutrition, 9(5), 533-43. doi: 10.1093/advances/nmy035.

Neavin, D.R., Liu, D., Ray, B., \& Weinshilboum, R.M. (2018). The role of the aryl hydrocarbon receptor(AHR) in immune and inflammatory diseases. International Journal of Molecular Sciences, 19(12), 3851. doi: 10.3390/ijms 19123851.

Netland, J., Meyerholz, D.K., Moore, S., Cassell, M., \& Perlman, S. (2008). Severe acute respiratory syndrome coronavirus infection causes neuronal death in the absence of encephalitis in mice transgenic for human ACE-2. Journal of Virology, 82(15), 7264-75. doi: 10.1128/JVI.00737-08.

O’Driscoll, C.A., \& Mezrich, J.D. (2018). The aryl hydrocarbon receptor as an immune-modulator of atmospheric particulate matter-mediated autoimmunity. Frontiers in Immunology, 9(2833). doi: 10.3389/fimmu.2018.02833.

Okey, A.B. (2007). An aryl hydrocarbon receptor odyssey to the shores of toxicology: the Deichmann lecture. Toxicological Sciences, 98(1), 5-38. doi: 10.1093/toxsci/kfm096.

Opitz, C.A., Litzenburger, U.M., Sahm, F., Ott, M., Tritschler, I., Trump, S., Schumacher, T., Jestaedt, L., Schrenk, D., Weller, M., Jugold, M., Guillemin, G.J., Miller, C.L., Lutz, C., Radlwimmer, B., Lehmann, I., von Deimling, A., Wick, W., \& Platten, M. (2011). An endogenous tumour-promoting ligand of the human aryl hydrocarbon receptor. Nature, 478(7368), 197-203. doi: 10.1038/nature10491.

Ott, M., Litzenburger, U.M., Rauschenbach, K.J., Bunse, L., Ochs, K., Sahm, F., Pusch, S., Opitz, C.A., Blaes, J., von Deimling, A., Wick, W., Platten, M. (2015). Suppression of TDOmediated tryptophan catabolism in glioblastoma cells by a steroid-responsive FKBP52-dependent pathway. Glia, 63(1), 78-90. doi: 10.1002/glia.22734.

Oxley, T.J., Mocco, J., Majidi, S., Kellner, C.P., Shoirah, H., Singh, I.P., De Leacy, R.A., Shigematsu, T., Ladner, T.R., Yaeger, K.A., Skliut, M., Weinberger, J., Dangayach, N.S., Bederson, J.B., Tuhrim, S., \& Fifi, J.T. (2020). Largevessel stroke as a presenting feature of Covid-19 in the young. New England Journal of Medicine, 382(20), e60. doi:10.1056/NEJMc2009787.

Román, G.C., Spencer, P.S., Reis, J., Buguet, A., Faris, M.E.A., Katrak, S.M., Láinez, M., Medina, M.T., Meshram, C., Mizusawa, H., Öztürk, S., \& Wasay, M. (2020). The neurology 
of COVID-19 revisited: A proposal from the Environmental Neurology Specialty Group of the World Federation of Neurology to implement international neurological registries. Journal of Neurological Sciences, 414, 116884. doi: 10.1016/j.jns.2020.116884.

Rothhammer, V., \& Quintana, F.J. (2019). The aryl hydrocarbon receptor: an environmental sensor integrating immune responses in health and disease. Nature Reviews Immunology, 19(3), 184-97. doi: 10.1038/s41577-019-0125-8.

Rzemieniec, J., Wnuk, A., Lasoń, W., Bilecki, W., \& Kajta, M. (2019). The neuroprotective action of 3,3'-diindolylmethane against ischemia involves an inhibition of apoptosis and autophagy that depends on HDAC and AhR/CYP1A1 but not ER $\alpha / C Y P 19 A 1$ signaling. Apoptosis, 24, 435-52. doi:org/10.1007/s10495-019-01522-2.

Sedlmayr, P. (2007). Indoleamine 2,3-dioxygenase in maternofetal interaction. Current Drug Metabolism, 8(3), 205-8. doi: $10.2174 / 138920007780362491$.

Stapelberg, M., Zobalova, R., Nguyen, M.N., Walker, T., Stantic, M., Goodwin, J., Pasdar, E.A., Thai, T., Prokopova, K., Yan, B., Hall, S., de Pennington, N., Thomas, S.R., Grant, G., Stursa, J., Bajzikova, M., Meedeniya, A.C., Truksa, J., Ralph, S.J., Ansorge, O., Dong, L.F., \& Neuzil, J. (2014). Indoleamine-2,3-dioxygenase elevated in tumorinitiating cells is suppressed by mitocans. Free Radical Biology \& Medicine, 67, 41-50. doi:10.1016/j.freeradbiomed. 2013.10.003.

Stevens, E.A., Mezrich, J.D., \& Bradfield, C.A. (2009) The aryl hydrocarbon receptor: a perspective on potential roles in the immune system. Immunology, 127(3), 299-311. doi: 10.1111/j.1365-2567.2009.03054.x.

Stockinger, B., Di Meglio, P., Gialitakis, M., \& Duarte, J.H. (2014). The aryl hydrocarbon receptor: Multitasking in the immune system. Annual Review of Immunology, 32, 403-32. doi: 10.1146/annurev-immunol-032713-120245.

Stockinger, B., Hirota, K., Duarte, J., \& Veldhoen, M. (2011). External influences on the immune system via activation of the aryl hydrocarbon receptor. Seminars in Immunology, 23(2), 99-105. doi: 10.1016/j.smim.2011.01.008.

Subramanian, H.H., \& Holstege, G. (2013). Stimulation of the midbrain periaqueductal gray modulates preinspiratory neurons in the ventrolateral medulla in the rat in vivo. Journal of Comparative Neurology, 521(13), 3083-98. doi: 10.1002/cne.23334.

Takami, M., Fujimaki, K, Nishimura, M., \& Iwashima, M. (2015). AhR is a molecular target of calcitriol in human T cells. Journal of Immunology, 195(6), 2520-23. doi:10.4049/jimmunol.1500344.

Tsatsakis, A., Petrakis, D., Nikolouzakis, T.K., Docea, A.O., Calina, D., Vinceti, M., Goumenou, M., Kostoff, R.N., Mamoulakis, C., Aschner, M., \& Hernández, A.F. (2020). COVID-19, an opportunity to reevaluate the correlation between long-term effects of anthropogenic pollutants on viral epidemic/pandemic events and prevalence. Food and Chemical Toxicology, 14(111418). doi: 10.1016/j.fct.2020.111418

Veldhoen, M., Hirota, K., Westendorf, A.M., Buer, J., Dumoutier, L., Renauld, J.C., \& Stockinger, B. (2008). The aryl hydrocarbon receptor links TH17-cell mediated autoimmunity to environmental toxins. Nature, 453(7191), 106-9. doi: 10.1038/nature06881.

Vrzal, R., Stejskalova, L., Monostory, K., Maurel, P., Bachleda, P., Pavek, P., \& Dvorak, Z. (2009). Dexamethasone controls aryl hydrocarbon receptor (AhR)-mediated CYP1A1 and CYP1A2 expression and activity in primary cultures of human hepatocytes. Chemical and Biological Interactions, 179, 288-96. doi: 10.1016/j.cbi.2008.10.035.

Weidenbusch, M., Rodler, S., Song, S., Romoli, S., Marschner, J.A., Kraft, F., Holderied, A., Kumar, S., Mulay, S.R., Honarpisheh, M., Kumar Devarapu, S., Lech, M., \& Anders, H.J. (2017). Gene expression profiling of the Notch-AhR-IL22 axis at homeostasis and in response to tissue injury. Bioscience Reports, 37(6), BSR20170099. doi.org/10.1042/BSR20170099.

Wheeler, M.A., Rothhammer, V., \& Quintana, F.J. (2017). Control of immune-mediated pathology via the aryl hydrocarbon receptor. Journal of Biological Chemistry, 292(30), 12383-9. doi: 10.1074/jbc.R116.767723.

Wichmann, D., Sperhake, J.-P., Lütgehetmann, M., Steurer, S., Edler, C., Heinemann, A., Heinrich, F., Mushumba, H., Kniep, I., Schröder, A.S., Burdelski, C., de Heer, G., Nierhaus, A., Frings, D., Pfefferle, S., Becker, H., Bredereke-Wiedling, H., de Weerth, A., Paschen, H.R., Sheikhzadeh-Eggers, S., Stang, A., Schmiedel, S., Bokemeyer, C., Addo, M.M., Aepfelbacher, M., Püschel, K., \& Kluge, S. (2020). Autopsy findings and venous thromboembolism in patients with COVID-19:A prospective cohort study. Annals of Internal Medicine, M202003. doi: 10.7326/M20-2003.

Xie, H.Q., Xu, H.-M., Fu, H.-L., Hu, Q., Tian, W.-J., Pei, X.-H., \& Zhao, B. (2013). AhR-mediated effects of dioxin on neuronal acetylcholinesterase expression in vitro. Environmental Health Perspectives, 121, 613-18. doi:org/10.1289/ehp.1206066.

Xu, Z., Shi, L., Wang, Y., Zhang, J., Huang, L., Zhang, C., Liu, S., Zhao, P., Liu, H., Zhu, L., Tai, Y., Bai, C., Gao, T., Song, J., Xia, P., Dong, J., Zhao, J., \& Wang, F.S. (2020). Pathological findings of COVID-19 associated with acute respiratory distress syndrome. Lancet Respiratory Medicine, 8(4), 420-2. doi: 10.1016/S2213-2600(20)30076-X.

Yan, J., Guo, J., Fan, C., Juan, J., Yu, X., Li, J., Feng, L., Li, C., Chen, H., Qiao, Y., Lei, D., Wang, C., Xiong, G., Xiao, F., He, W., Pang, Q., Hu, X., Wang, S., Chen, D., Zhang, Y., Poon, L.C., \& Yang, H. (2020). Coronavirus disease 2019 (COVID-19) in pregnant women: A report based on 116 cases. American Journal of Obstetrics and Gynecology, S0002-9378(20)30462-2. doi: 10.1016/j.ajog.2020.04.014.

Ye, Z., Zhang, Y., Wang, Y., Huang, Z., \& Song, B. (2020). Chest CT manifestations of new coronavirus disease 2019 (COVID-19): a pictorial review. European Radiology, 1-9. doi:10.1007/s00330-020-06801-0.

Yi, S.-W., Hong, J.-S., Ohrr, H., \& Yi, J.J. (2014). Agent orange exposure and disease prevalence in Korean Vietnam veterans: the Korean veterans health study. Environmental Research, 133, 56-65. doi: org/10.1016/j.envres.2014.04.027.

Zhang, C., Shi, L., \& Wang, F.S. (2020). Liver injury in COVID-19: management and challenges. Lancet Gastroenterology and Hepatology, 5(5), 428-30. doi: 10.1016/S24681253(20)30057-1. 
Zheng, Y.Y., Ma, Y.T., Zhang, J.Y., \& Xie, X. (2020). COVID-19 and the cardiovascular system. Nature Reviews Cardiology, 17(5), 259-60. doi:10.1038/s41569-020-0360-5.

Zhou, B., Wang, X., Li, F., Wang, Y., Yang, L., Zhen, X., \& Tan, W. (2017). Mitochondrial activity and oxidative stress functions are influenced by the activation of AhR-induced CYP1A1 overexpression in cardiomyocytes. Molecular Medicine Reports, 16(1), 174-80. doi: 10.3892/mmr.2017.6580.

Zhou, L., Zhang, M., Wang, J., \& Gao, J. (2020) SarsCov-2: Underestimated damage to nervous system. Travel Medicine and Infectious Disease. doi.org/10.1016/j.tmaid. 2020.101642 .

Zubair, A.S., McAlpine, L.S., Gardin, T., Farhadian, S., Kuruvilla, D.E., Spudich, S. (2020) Neuropathogenesis and neurologic manifestations of the coronaviruses in the age of coronavirus disease 2019. JAMA Neurology. doi:10.1001/jamaneurol.2020.2065.

\section{Note}

\section{Data mining on the AhR and IDOI genes}

We conducted a gene expression data mining aimed to identify conditions under which AhR and IDO1 undergo simultaneous upregulation. When the search was limited to human lung-derived samples, we confirmed that increased expression of AhR and IDO1 genes occurred in response to viral infections, including SARS-CoV-2 and respiratory syncytial virus (RSV) (Fig. 3A). This was accompanied by elevation of proinflammatory gene expression, activation of the TF/PAI-1 signaling pathway, and upregulation of CYP1A1 (Fig. 3A).

In parallel, we investigated drugs in clinical use for propensity of affecting the expression of both AhR and IDO1 genes by means of integrative analysis of bioinformatics data collected on human and non-human tissues to discover how the suspected key drivers of infection mediated by $\mathrm{CoV}$ and perhaps by SARS-CoV-2, AhR and IDO1, can be affected by medications already employed to treat other human diseases.
Surprisingly, there are licensed drugs possessing such dual antagonistic properties (Fig. 3B) such as rosiglitazone (antidiabetic), dexamethasone and betamethasone (glucocorticoids with broad range uses including asthma, chronic obstructive pulmonary disease and rheumatoid arthritis), infliximab (TNF- $\alpha$ inhibitor), isoniazid (antituberculotic), niacinamide (vitamin $B_{3}$, dietary supplement), levonorgestrel (progestogen), promazine (neuroleptic), curcumin (herbal dietary supplement), lidocaine (local anesthetic and antiarrhythmic), and amitriptyline (antidepressant and anxiolytic). Calcitriol (vitamin $\mathrm{D}_{3}$ ) and fluticasone (steroid used for therapy of asthma and chronic obstructive pulmonary disease), preferentially down-regulated the AhR gene in the context of this analysis (Fig. 3B).

No clinical data supporting use of any medications listed in Fig. 3B in therapy or prevention of $\mathrm{CoV}$ infections currently exist, nor is it known if any, how, and in which phase of the disease these medications can be recommended for use. Rigorous clinical trials are necessary before any recommendation regarding future use of these medications in therapy of $\mathrm{CoV}$ infections, including Covid-19, can be made.

Blanco-Melo, D., Nilsson-Payant, B.E., Liu, W.C., Uhl, S., Hoagland, D., Møller, R., Jordan, T.X., Oishi, K., Panis, M., Sachs, D., Wang, T.T., Schwartz, R.E., Lim, J.K., Albrecht, R.A., \& ten Oever, B.R. (2020). Imbalanced host response to SARS-CoV-2 drives development of COVID-19. Cell, 181(5), 1036-1045.e9, doi: 10.1016/j.cell.2020.04.026.

Hruz, T., Laule, O., Szabo, G., Wessendorp, F., Bleuler, S., Oertle, L., Widmayer, P., Gruissem, W., \& Zimmermann, P. (2008). Genevestigator v3: a reference expression database for the meta-analysis of transcriptomes. Advances in Bioinformatics, 2008: 420747, doi: 10.1155/2008/420747.

Kupershmidt, I., Su, Q.J., Grewal, A., Sundaresh, S., Halperin, I., Flynn, J., Shekar, M., Wang, H., Park, J., Cui, W., Wall, G.D., Wisotzkey, R., Alag, S., Akhtari, S., \& Ronaghi, M. (2010). Ontology-based meta-analysis of global collections of high-throughput public data. PLoS One, 5(9):e13066, doi: 10.1371/journal.pone.0013066. 
A

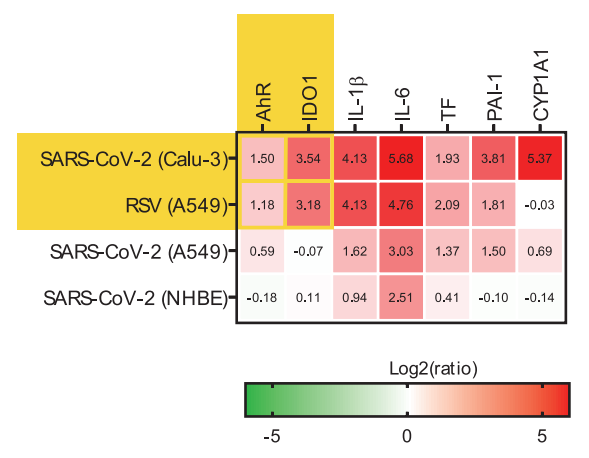

B

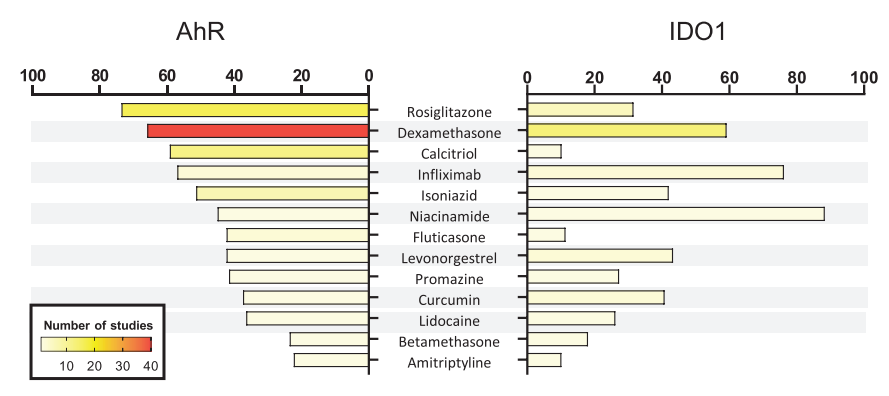

Fig. 3. Data mining on the modulation of AhR and IDO1 genes. (A) Conditions under which both AhR and IDO1 genes undergo upregulation were identified using biclustering tool of Genevestigator 7.6.0 (www.genevestigator.com) (Hruz et al., 2008). Query was limited to mRNAseq data from human lung tissues and cell lines. The results are marked with yellow background. It was revealed that significant up-regulation of $A H R$ and $I D O 1$ occurred in the lung adenocarcinoma cell line Calu- 3 samples infected with SARS-CoV-2 (USA-WA1/2020 isolate) at a MOI (multiplicity of infection) of 2 and harvested $24 \mathrm{~h}$ post-infection (hpi) versus mock-infected Calu- 3 cells. Similar observation was made in human lung adenocarcinoma cell line A549 samples infected with respiratory syncytial virus (RSV) A2 strain (MOI 2; 24 hpi) in comparison to mock control (Blanco-Melo et al., 2020). There were no other experimental results demonstrating concurrent upregulation of $A H R$ and IDO1 in the human lung in the Genevestigator database encompassing 2536 mRNAseq studies. Initial heatmap (yellow background) was extended with data on the expression profile of genes driving the AhR-dependent pathologies: inflammation, thrombosis, and fibrosis (Fig. 2). The specific genes code for (1) proinflammatory interleukin $1 \beta$ (IL-1 $\beta$ ) and interleukin 6 (IL-6); (2) thromboembolism-related tissue factor (TF) and plasminogen activator inhibitor-1 (PAI-1); and (3) fibrosis-related P450 cytochrome 1A1 (CYP1A1). Data from A549 and Normal Human Bronchial Epithelial (NHBE) cells infected with SARS-CoV-2 (MOI 2, 24 hpi) were provided for reference. (B) Medications modulating the expression of AhR and IDO1 genes were identified using Pharmaco Atlas of the BaseSpace Correlation Engine software suite (www.nextbio.com) (Kupershmidt et al., 2010). A p-value significance cutoff of 0.05 (without any multiple testing correction) and a minimum absolute fold-change cutoff of 1.2 were used by the software for identification of medications affecting expression of the genes of interest. Then, grading of significant factors was conducted based on the following conditions: (1) total number of medication specific studies in which the gene was measured; (2) number of medication-specific studies in which the gene's expression was found to be significantly altered; (3) gene's degree of down- or up-regulation in comparison to all other genes with altered expression within each of the medication studies; (4) consistency of the gene's association across the medication studies. Additional statistical criteria were applied, such as correction for multiple hypothesis testing, to finally rank the medications. The most significant result was assigned with a numerical score of 100 , and the other medications' scores were normalized to the top-ranked result. Analyses were performed separately for AhR and IDO1 genes. Only medications significantly downregulating both genes were plotted. Numbers of studies are shown by means of the color scale ranging from light yellow $(n=1)$ to dark red $(n=40)$. 